\title{
Status and results from the ANTARES and KM3NeT-ARCA neutrino telescopes
}

\author{
Paolo Fermani*i \\ Sapienza Università di Roma - INFN Sezione di Roma, P.le Aldo Moro 5, 00185, Roma, Italy. \\ E-mail: paolo.fermani@roma1.infn.it
}

\begin{abstract}
ANTARES, currently the largest neutrino telescope in the Mediterranean Sea, has been operating for more than 10 years. ANTARES provides unprecedented sensitivity for neutrino source searches in the Southern Sky at TeV energies, so that valuable constraints can be set on the origin of the cosmic neutrinos discovered by the IceCube detector. ANTARES has also constrained the neutrino emission from possible Dark Matter annihilation in massive objects like the Sun or the Galactic Centre, and has measured the neutrino oscillation parameters in the atmospheric sector. Building on the ANTARES experience, KM3NeT, a new, much larger detector with improved design and technology is under construction on two sites in the Mediterranean sea. Deployed off the coast of Sicily, the ARCA telescope (Astroparticle Research with Cosmics in the Abyss) will be dedicated to the detection of high-energy astrophysical neutrinos. When completed, KM3NeTARCA detector with dimensions exceeding one kilometre cube, will reconstruct with an excellent angular resolution signatures of neutrinos of all flavors in a very clear deep-sea water environment. KM3NeT-ARCA, situated in the Northern hemisphere, will be able to observe up-going neutrinos from most of the Galactic Plane, making it possible to study the neutrino fluxes from different astrophysics sources, as well as neutrinos from Dark Matter annihilation. The latest results from ANTARES and the perspectives of the KM3NeT-ARCA detector will be presented.
\end{abstract}

The 21st international workshop on neutrinos from accelerators (NuFact2019)

August 26 - August 31, 2019

Daegu, Korea

* Speaker.

${ }^{\dagger}$ on behalf of the ANTARES and KM3NeT collaborations 


\section{The ANTARES neutrino telescope}

ANTARES is the largest underwater neutrino telescope operating since 2007 in the Northern hemisphere [1]. It is located in the Mediterranean Sea, $40 \mathrm{~km}$ off the French coast at a depth of $2475 \mathrm{~m}$. It is a 3-dimensional array of 885 photomultipliers distributed in 25 storeys along 12 vertical lines. Its aim is to detect high-energy neutrinos up to $10^{4} \mathrm{TeV}$. The detection is based on the measurement of Cherenkov light emitted by charged leptons: muons produced in $v_{\mu} \mathrm{CC}$ interactions with the matter in the surrounding of the telescope propagate through the detector as track-like events, while $v_{e}$ and $v$ NC interactions cause hadronic shower-like events. An excellent angular resolution of $\simeq 0.4^{\circ}$ for tracks of muon neutrinos above a few $\mathrm{TeV}$ and $3^{\circ}$ for showers is achieved.

\section{Neutrino oscillations and sterile neutrino analysis}

The energy threshold of the ANTARES neutrino telescope is $\simeq 20 \mathrm{GeV}$ : enough to allow ANTARES to be sensitive to the first atmospheric neutrino oscillation minimum, making it possible to study the atmospheric muon neutrino disappearance due to neutrino oscillations. Data collected by ANTARES from 2007 to 2016 (2830 days of lifetime) have been used to measure the parameters $\Delta m_{32}^{2}$ and $\theta_{23}$ [2]. In this analysis only track events have been selected, the other event topologies are treated as background. Events have been reconstructed using two different algorithms while the muon range has been used for the energy estimation. A set of selection criteria has been applied resulting in a total of 7710 events. A binned likelihood fit has been performed in two dimensions: the reconstructed energy and the cosine of the track zenith angle. A complete analysis of the systematics has also been carried out. The results, shown in figure 1a, discard the non-oscillation hypothesis with a significance of $4.6 \sigma$. Since the addition of a sterile neutrino is expected to cause visible effects in the same energy and zenith interval of the oscillation analysis, the same procedure and data sample has been used to constrain the $3+1$ neutrino model parameters, particularly the mixing angles $\theta_{24}$ and $\theta_{34}$. The results are summarized in figure $1 \mathrm{~b}$.

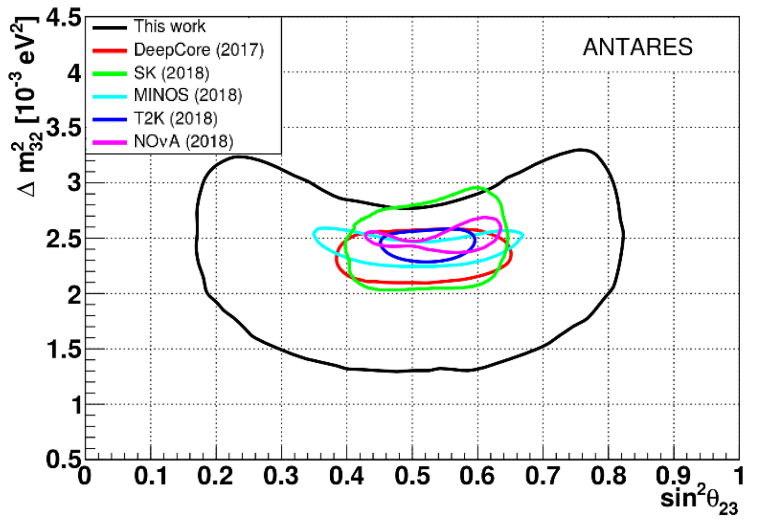

(a)

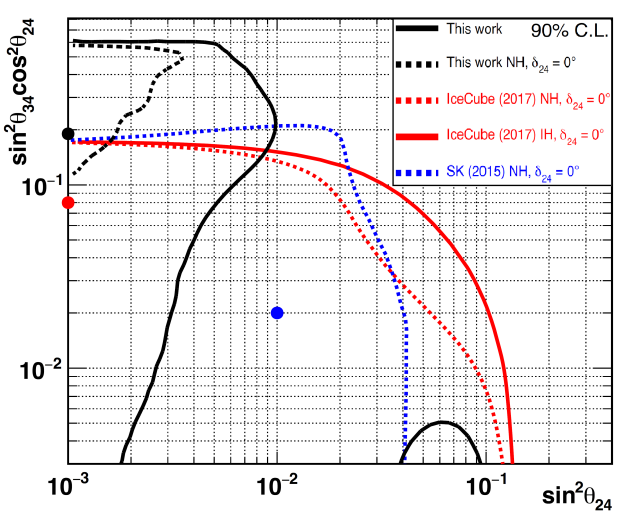

(b)

Figure 1: (a) $90 \%$ C.L. contours in the $\Delta m_{32}^{2}, \theta_{23}$ oscillation space; (b) $90 \%$ C.L. limits in the sterile neutrino $\theta_{34}, \theta_{24}$ space [2]. Both ANTARES limits (black curves) are compared with the limits from other experiments. 


\section{Neutrinos from astrophysical point sources}

The observation of neutrinos from cosmic sources has been the main objective of the ANTARES experiment. ANTARES has a very good visibility of the Southern sky and of the Galactic plane and an optimal angular resolution. The results of the search for cosmic point-like neutrino sources are presented. Track-like and shower-like events have been used, collected during 11 years of ANTARES data-taking (2007-2017) corresponding to a total livetime of 3136 days [3]. The event selection criteria have been optimised to minimise the neutrino flux needed for a $5 \sigma$ discovery of a point-like source emitting with a spectrum $\Phi(E) \propto E^{-2}$. The final sample of events used in the analysis comprises 8754 tracks and 195 showers. Possible clusters of cosmic neutrinos from point-like sources have to be identified over the background of randomly distributed atmospheric neutrinos. For this purpose an unbinned method based on an extended maximum likelihood ratio test statistic is employed. Three different searches for astrophysical neutrino sources have been performed: a scan over the whole ANTARES visible sky (see figure 2a), a survey of 112 astrophysical candidates and an investigation of 75 IceCube tracks. The resulting limit is reported in figure $2 \mathrm{~b}$.

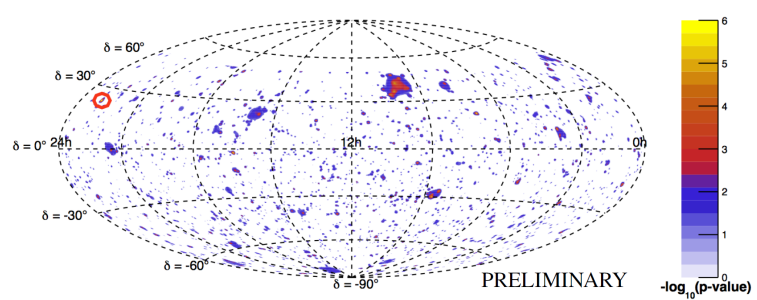

(a)

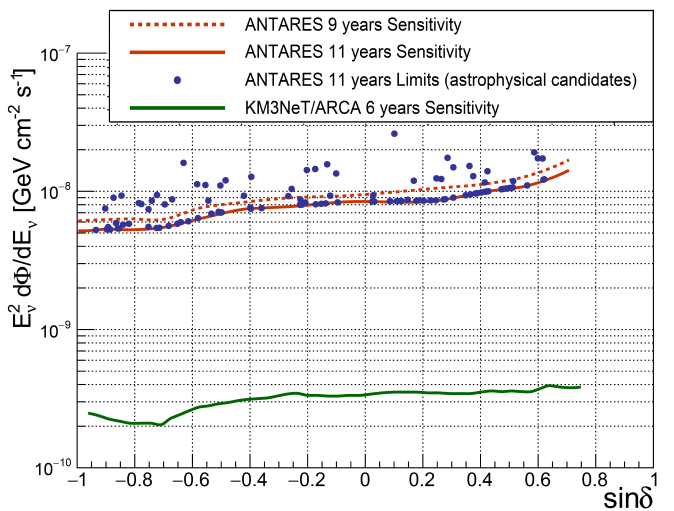

(b)

Figure 2: (a) Sky map in equatorial coordinates of clusters in the ANTARES visible sky. Red contour indicates the location of the most significant one: Blazar MG3 J225517+2409 seen with an excess of $1.2 \sigma$; (b) $90 \%$ C.L. upper limits on the signal flux from the investigated astrophysical candidates vs. the source declination. The KM3NeT/ARCA 6 years sensitivity is also shown [3].

\section{Neutrinos from the cosmic diffuse flux}

IceCube observed a high energy diffuse flux of cosmic neutrinos that cannot be associated to any point source. This flux can be explained considering a spectrum with two components: one soft and diffuse of Galactic origin and a harder one coming from extragalactic sources. ANTARES also observed an excess of events over the expected atmospheric backgrounds which is consistent with the IceCube observation. The analysis presented here is based on the data collected over an 11 years period by ANTARES (2007-2018) corresponding to a total livetime of 3380 days [4]. The signal flux has been described by an unbroken single power law energy spectrum: $d \phi / d E \propto E^{-\Gamma}$. 
In this analysis both track and shower events have been selected, resulting in a final sample of 50 possible neutrino candidates ( 27 tracks and 23 showers). For the background expectation, Monte Carlo events have been simulated assuming the Honda model for the conventional component and the Enberg et al. model for the prompt component. Resulting in $36.1 \pm 8.7$ events. Thus an excess of $1.8 \sigma$ has been observed in the data above the expectation from atmospheric backgrounds (see figure 3). This excess is compatible with the hypothesis of an isotropically distributed flux over the whole sky (with equipartition between the three neutrino flavours). The excess of events has been fitted with a maximum likelihood procedure. For the cosmic flux the best fit parameters are: $\Gamma=2.3 \pm 0.4$ for the spectral index and $\Phi(100 \mathrm{TeV})=(1.51 \pm 1.0) \times 10^{-18}\left(\mathrm{GeV} \cdot \mathrm{cm}^{2} \cdot \mathrm{sr} \cdot \mathrm{s}\right)^{-1}$ for the flux. The null-cosmic contribution in the high-energy sample has been excluded at $90 \%$ C.L..
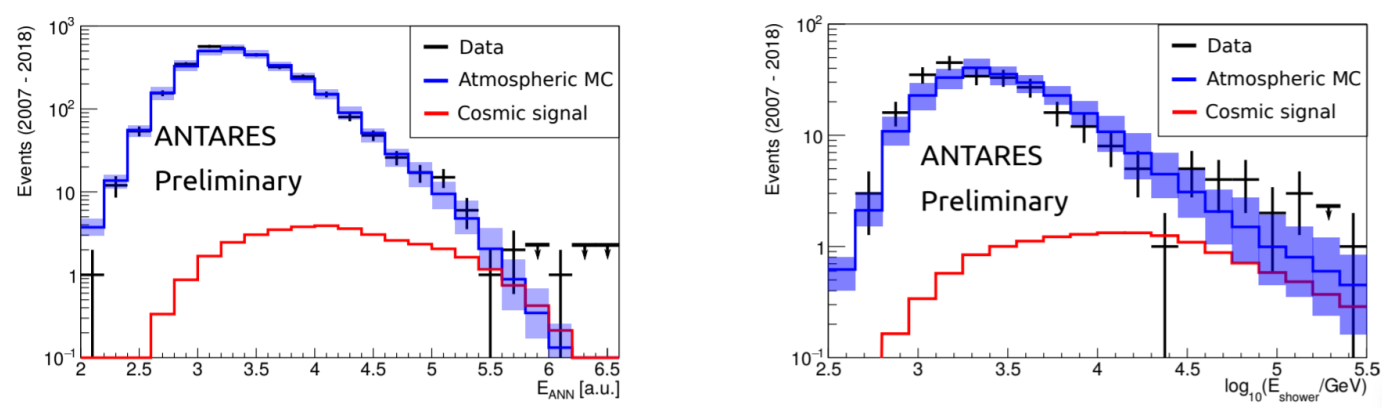

Figure 3: Energy estimator distributions for the track (left) and the shower (right) channels for the events selected. The data (black points), the cosmic neutrino expectation (red histogram) and the contribution from atmospheric events (blue histogram, band indicates the uncertainty) [4] are shown.

\section{Neutrinos from Dark Matter annihilation in the Galactic Centre}

An important result that can be achieved with neutrino telescopes is the indirect identification of Dark Matter particles signature. One of the most common candidate as DM particle is the WIMP (Weakly Interacting Massive Particle). WIMPs are present into the galactic halo and accumulate into the Galactic Centre (GC) where they can self-annihilate producing Standard Model secondary particles, like bosons and quarks, that can eventually decay producing neutrinos. In the analysis presented here [5], the following channels have been studied: $b \bar{b}, \tau^{+} \tau^{-}, W^{+} W^{-}, \mu^{+} \mu^{-}, \nu \bar{v}$. For each channel the considered neutrino energy spectra (i.e. the number of neutrinos emitted per energy and per WIMP annihilation) depend on the WIMP mass. The spectra refer to an isotropic emission of neutrinos at the source, modulated to account for flavour oscillation between source and detection point in the long baseline approximation. The predictions on neutrino fluxes from DM annihilation depend on the distribution of the DM density. For this the Navarro-Frank-White and Einasto parametrisations have been used. 11 years of data taken with the ANTARES neutrino telescope (2007-2017) corresponding to a total lifetime of 3170 days have been analysed. From the relation between the observed neutrino flux and the DM annihilation rate a measurement of the integrated neutrino and antineutrino flux from the GC can be converted into limits on the thermally averaged DM annihilation cross-section. These estimated upper limits are shown in figure $4 \mathrm{a}$ and $4 \mathrm{~b}$. 


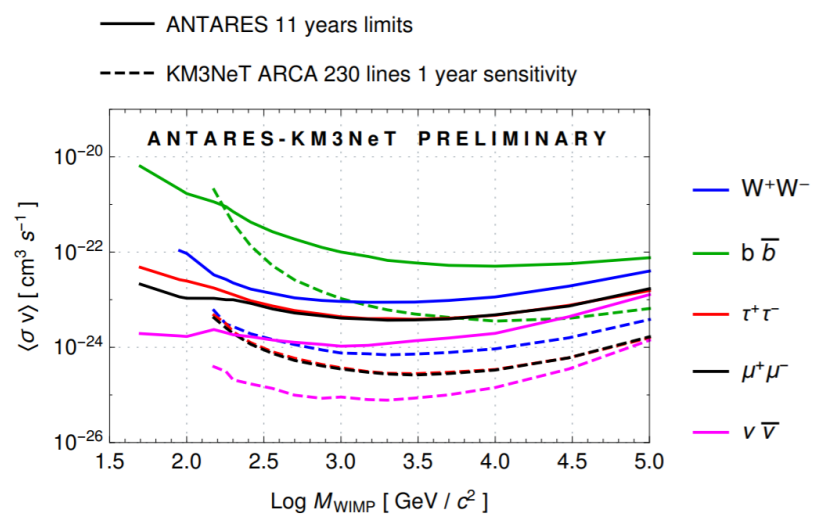

(a)

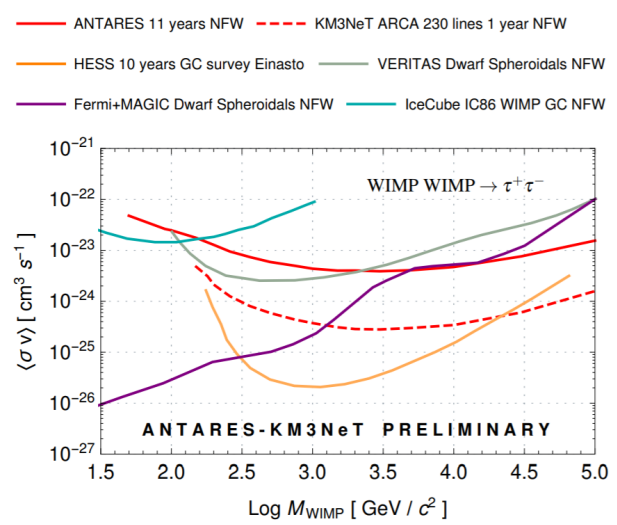

(b)

Figure 4: (a) 90\% C.L. upper limits on $v_{\mu}+\bar{v}_{\mu}$ flux converted in limits on the thermally averaged cross-section for WIMP annihilation (NFW profile); (b) $90 \%$ C.L. upper limits for the $\tau^{+} \tau^{-}$channel compared with other experiments. The sensitivity for the KM3NeT-ARCA detector is also reported for 1 year of observation [5].

\section{The future of underwater Cherenkov neutrino telescopes: KM3NeT-ARCA}

KM3NeT is a network of submarine Cherenkov neutrino detectors under construction in two different sites in the Mediterranean Sea [6]. ARCA, the $\mathrm{km}^{3}$ detector at the Italian site, close to the Sicilian coast will be devoted to the detection of high energy ( $\mathrm{TeV}-\mathrm{PeV})$ astrophysical neutrinos. The telescope is an array of flexible strings anchored to the sea floor and kept close to vertical by submerged buoys. The strings are instrumented with digital optical modules (DOMs), hosted within pressure-resistant glass spheres, each housing 31 3-inches photomultiplier tubes (PMTs) and the readout electronics. The geometry of the detectors has been adapted to their physics goals. ARCA strings (placed at $3500 \mathrm{~m}$ depth), are $\sim 700 \mathrm{~m}$ high and are equipped with 18 DOMs, with a vertical spacing of $36 \mathrm{~m}$. The distance between strings is $\sim 90 \mathrm{~m}$. The KM3NeT DOM design, provides optimised directional information. The first two lines of ARCA have been deployed in December 2015 and May 2016 respectively. It is planned to have 6 more strings deployed in the summer 2020. An angular resolution of the order of $0.1^{\circ}$ is expected for the ARCA detector, when completed. The first ARCA data has been analysed and the first neutrino candidates have been identified. This indicates the robustness of the detector calibration, the validity of the simulation chain and overall a good understanding of the detector response [7].

\section{References}

[1] M. Ageron et al. (ANTARES collaboration), NIM A 656, 11-38 (2011).

[2] A. Albert et al. (ANTARES collaboration), JHEP 6, 113 (2019).

[3] G. Illuminati et al. (ANTARES collaboration), 36th ICRC, PoS 920 (2019).

[4] L. Fusco et al. (ANTARES collaboration), 36th ICRC, PoS 891 (2019).

[5] S. Gozzini et al. (ANTARES and KM3NeT collaborations), 36th ICRC, PoS 552 (2019).

[6] S. Adrian-Martinez et al. (KM3NeT collaboration), J. Phys. G 43 no.8, 084001 (2016).

[7] R. Coniglione et al. (ANTARES and KM3NeT collaborations), 36th ICRC, PoS 006 (2019). 